\title{
OPTN:a promising target of autophagy to treat autophagy defective diseases
}

\author{
Yueping Qiu ${ }^{1}$, Jiajia Wang ${ }^{1}$, Jincheng Wang ${ }^{1}$, Bo Yang ${ }^{1}$, Qiaojun $\mathrm{He}^{1}$, and Qinjie Weng ${ }^{1}$ \\ ${ }^{1}$ Zhejiang University - Zijingang Campus
}

May 11, 2020

\begin{abstract}
Optineurin (OPTN) is a multifunctional adaptor protein that mainly involved in autophagy. While it was defined as an autophagy receptor and inducer, the extensive and important role in autophagy is hardly mentioned. What's more, there are some critical disorders are closely related to OPTN, such as Glaucoma, Amyotrophic lateral sclerosis, Paget's disease of bone, Crohn's disease, cancer and diabetic nephropathy. This review will depict the OPTN involved autophagic process, from the recognition of cargo to the degradation in the autolysosome in molecular terms, and in particular highlight the process that compromised in disease related to OPTN. And we looking forward to strategies to modulate OPTN specifically with no toxic effects so that establish an efficient therapy for autophagy defective diseases.
\end{abstract}

\section{Abbreviations list:}

$\mathrm{NF}-x \mathrm{~B}$ nuclear factor- $x \mathrm{~B}$

TNF- $\alpha$ tumour necrosis factor- $\alpha$

ROS reactive oxygen species

mRNA Nucleic acids \& related terms

LPS lipopolysaccharide

PI3K phosphoinositide 3-kinase

\section{Introduction}

The lysosomal degradation pathway of autophagy is a highly conserved mechanism in eukaryotic cells and play an important role in cellular, tissue and organismal homeostasis by removing dysfunctional organelles, intracellular bacterial and aggregated proteins selectively (Zaffagnini et al. , 2016). Autophagy can be activated by cellular stress such as starvation, hypoxia, oxidative stress, protein aggregation, endoplasmic reticulum (ER) stress and others, which triggers the cell survival process and thus provides energy to the cells when energy is consumed (Levineet al. , 2019). Thus, autophagy is generally considered to be a cell survival process and play an important role in the maintenance of cellular homeostasis.

At present, it is divided into three categories according to the occurrence process: Macroautophagy (also termed autophagy), Microautophagy, and Chaperone-mediated autophagy (CMA), the following refers to the first category unless otherwise specified (Wen et al. , 2016). It is generally accepted that autophagy is a more selective process than originally expected. Autophagy can be divided into different types, according to the different autophagy substrates(also called cargo), for example, aggrephagy for protein aggregates, lysophagy for damaged lysosomes, mitophagy for mitochondria and xenophagy for intracellular bacterial. Conservatively, most of these different types of selective autophagy pathways use a common mechanism. 
Up to now, there are 34 Autophagy-related (ATG) genes that is discovered in yeast and 15 of which are "core" ATG genes that are commonly necessary to the different pathways of autophagy (Nakatogawa et al. , 2009). The autophagosome biogenesis process can be divided into four steps, the first is the nucleation and expansion of the phagophore, the second is the formation of autophagosome, the third is the autophagosome and lysosome fuse to form autolysosome, and the last is degrades the cargos by enzymes in lysosomes.

It is also generally conceded that the trigger of autophagy results in ATGs recruitment, Unc-51-like kinase 1 (ULK1) complex (consisting of ULK1/2, ATG13, FIP200 and ATG101), to the phagophore assembly site (PAS), a specific subcellular location where the nucleation of phagophore to be triggered. Then a cup-shaped structure isolation membrane is formed from the different source of membrane including mitochondria, endoplasmic reticulum. Meanwhile, 'eat-me' signals are necessary to cargo to be selectively recognized by several autophagy receptors that link the cargo to the autophagic membrane via their light chain 3 (LC3)-interacting region (LIR) by Atg8. The most primarily 'eat-me' signals is ubiquitin (Ub) chains. Atg8 is a ubiquitin-like protein, can be tightly bound to the autophagic membranes when it was cleaved at its C-termini by the ATG4 pro- teases to expose a C-terminal glycine producing the form I of the ATG8 molecule, and then conjugated to PE and being an PE-conjugated form II of ATG8 proteins. Yeast only owns the one kind of Atg8 protein, while mammals have at least seven if not more ATG8 proteins that can be fell into two subfamilies containing not less than three MAP1 light chain 3 (LC3A, B and C) and four gamma-aminobutyrate receptor-associated protein (GABARAP) and GABARAP-like proteins (ATG8L/GEC-1/GABARAPL1, GATE-16/GABARAPL2 and GABARAPL3) (Xin et al. , 2001; He et al. , 2003).Many proteins have been identified as autophagy receptors that can recognize the 'eat-me' signals of cargos, and the classic and wildly accepted autophagy receptors including p62, OPTN, TAX1BP1, NDP52 (Pohl et al. , 2019). Each of these receptors contains LC3 interacting regions (LIRs) that facilitate their interaction with LC3-like molecules on the autophagosome (Wildet al. , 2011; Katsuragi et al. , 2015; Tumbarello et al. , 2015).

Furthermore, there is a clear etiological link between gene mutations that control autophagy and human diseases (especially neurodegenerative diseases, inflammatory diseases, and cancer) (Levine et al. , 2019). These diseases are largely untreatable and have no target for intervention that needed an interventional therapeutic target and an interventional target drug. Therefore, these genes that play an important role in autophagy have the opportunity to be the candidate therapeutic targets for these diseases. As we introduced above, the autophagy related genes mainly including Atgs and autophagy receptors. Among them, OPTN is well-documented to have a strong relationship with several disease, for example, neurodegenerative diseases, cancer, inflammatory diseases, etc . (Liu et al. , 2018; Weil et al. , 2018).

OPTN is a conservative protein in many species, including human, macaques, rats, pigs, and bovine and is widely in liver, heart, brain, placenta, liver, skeletal muscle, kidney, pancreas, retina, optic nerve blood vessels and so on. OPTN was identified as a negative regulator of NF- $\chi \mathrm{B}$ that competitively binds ubiquitin with NEMO (NF- $x \mathrm{~B}$ essential modulator) (Zhu et al. , 2007), and an autophagy receptor that connect the ubiquitinated autophagy substrate and LC3-positive autophagosome membrane (Wildet al. , 2011). What's more, OPTN is also an autophagy inducer that induce autophagic process upon overexpression (Ying et al. , 2016). Breakthrough has been made in comprehending the molecular mechanisms of OPTN in autophagy since 2011. OPTN is an important autophagy adaptor, participate in almost every step of the autophagy process and not just as a autophagy receptor. But the existing review neither clearly clarify this point neither sort out the existing literature lucidly.

Over the past decade of studies, OPTN has been involved in many biological process, and its role as autophagy receptor is a breakthrough finding which caught scientists attention in recent years. Contemporary, OPTN was closely associated with some incurable disease in human beings, such as ALS, glaucoma, Paget's disease, Crohn's disease, and recently cancer and diabetic nephropathy. However, the relationship between OPTN and these disease has not been well revealed and clarified. This review discusses the biological functions of OPTN in the process of autophagy and the perspective of autophagic mechanism that OPTN involved in several human diseases. 


\section{The Protein Structure and Cellular Function of OPTN}

Optineurin(OPTN) was first identified by a yeast two-hybrid screening in order to find interacting proteins of Ad E3 14.7-kDa protein (E3-14.7K) that inhibit TNF- $\alpha$ functions (Li et al. , 1998), and named as FIP-2(14.7K-interacting protein). Then Klaus et al . using "Data base searching" found a NEMO-related protein that shows a strong homology to NEMO, which is the second time that OPTN come into the sight of scientists and name NRP (the first letter of each word in "NEMO-related protein") (Schwamborn et al. , 2000). After then, more and more studies have sprung up like mushrooms that uncovering the enigma of OPTN in structure of protein, biological function and etiological link in disease.

The human OPTN protein contains 577 amino acids and is a $74 \mathrm{kD}$ scaffold protein contains several structural domains that endow its multiple abilities. And the mouseOptn gene codes for a 584-amino acid protein (67 $\mathrm{kD}$ ) which is $78 \%$ identical to human OPTN (Rezaie et al. , 2005). The public databases contain partial or complete sequences of OPTN homologues of macaques, rats, pigs, and bovine, all of which show a great extent of similarity to human OPTN, which means OPTN is a conserved gene that play an important role in vital activity. OPTN contains several structural domains and is consists of the structures including CC (coiled-coil) domain, LZ (leucine zipper) domain, LIR (LC3 interacting region) domain, UBAN (ubiquitinbinding domain (UBD) of ABIN proteins and NEMO) domain, ZF (zinc finger) domain. The LIR domain and UBD domain play an important role in autophagy and is the foundation of OPTN to function as an autophagy receptor. In detail, the LIR domain is the location that binding LC3II and the UBD domain is the place where attach the ubiquitinated cargos. The LC3II is an PE-conjugated form II of ATG8 proteins that can tightly bound to the autophagic membranes. So that, cargos are enclosed by autophagic membranes and turn into autophagosome and then degraded in autolysosome.

Many biological function of OPTN was reported so far. Firstly, Tayebeh et al. using immunocytochemistry to found the intracellular localization of OPTN and observed the colocalization with the Golgi apparatus (Rezaieet al. , 2002). A deeper study bring this colocalization to light, OPTN plays a vital role in the maintenance of Golgi integrity (Parket al. , 2006).Secondly, OPTN shows strong homology with NEMO, and was identified as a negative regulator of NF- $x \mathrm{~B}$ that competitively binds ubiquitin with NEMO, thus it may have role in NF- $x \mathrm{~B}$ signaling regulation. Thirdly, Wild et al. fully proved the specific interactions between OPTN and LC3/GABARAP proteins by pull-down assays in MCF-7 cells, yeast two-hybrid transformations, and purified proteins in vitro (Wild et al. , 2011). The vital role of OPTN in autophagy has attracted greater attention in the last ten years.

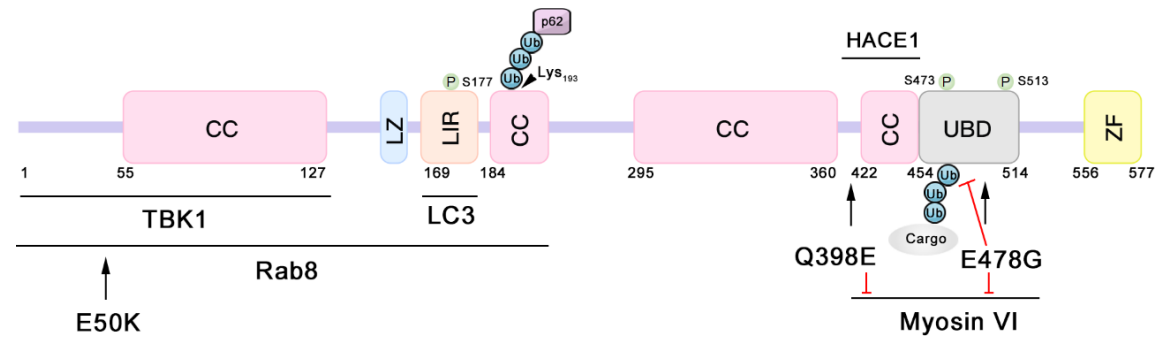

OPTN is widely expression in liver, heart, brain, placenta, liver, skeletal muscle, kidney, pancreas, retina, optic nerve blood vessels and so on. Many diseases have been reported to relevant to OPTN mutation or defective. Briefly, countless mutations have been reported in a subset of patients with Glaucoma or ALS. Moreover, GWAS have identify the OPTN as a genetic risk factor for Paget's disease of bone. Other diseases such as Cancer, Crohn's disease, Parkinson's disease, Alzheimer's disease, diabetic nephropathy, and neuronal intranuclear inclusion disease was related to OPTN to some degree. However, there is no compound can regulate OPTN directly so far.

FIGURE 1 Schematic representation of the domain of OPTN and several representative binding partners and mutations we reviewed below 


\section{OPTN is a key regulator in autophagy}

OPTN has been proposed to contribute to selective autophagy of depolarized mitochondria (mitophagy), protein aggregates (aggrephagy), and intracellular bacterial pathogens (xenophagy) through ubiquitin-signaling. What' more, OPTN has plenty of domains that mediate its interactions with several binding partners and it serves as a multifunctional adaptor for diverse autophagy events such as cargo recognition, autophagosome formation, autophagosome maturation, etc .

\subsection{The Role of OPTN in cargo recognition}

2.1.1 The recruitment of OPTN: As an autophagy receptor, OPTN act as a receptor for recognizing the ubiquitinated cargo, which form the molecular basis for selective autophagy. But before recognition the ubiquitinated cargo, OPTN should have to recruitment around the cargo first.

As examples on how OPTN are recruited around cargos we will discuss two different cargos: damaged mitochondria (mitophagy), and intracellular bacteria(xenophagy). In 2014, Wong et al . showed for the first time that Parkin mediates in stabilizing OPTN on the surface of damaged mitochondria, necessarily and sufficiently (Wong et al. , 2014). PINK1 (PTEN induced putative kinase 1) is a protein kinase that is mitochondria-targeted and parkin is a protein encoded by the PARK2 gene served as a ubiquitin E3 ligase. In the present of parkin in HeLa cells, OPTN is recruited to mitochondria right after parkin recruitment, and this recruitment could be stabilized via the UBAN domain probably due to its ubiquitin ligase activity. However, in the absence of parkin, OPTN puncta just localize to damaged mitochondria transiently and cannot even remain stably associated (Wong et al. , 2014). Straight after, another study reported that OPTN could dynamically recruited to damaged mitochondria via PINK1 to directly activate mitophagy independent of Parkin (Lazarou et al. , 2015). These two studies highlight that PINK1 might facility in OPTN recruitment and Parkin facility in stable OPTN in damaged mitochondria in mitophagy.

In a fashion similar to mitophagy, various intracellular bacterial pathogens are also targeted by cargo receptors for xenophagy via a ubiquitin-mediated pathway that surrounds bacteria with conjugated ubiquitin chains. At least four cargo receptors, namely NDP52, p62, NBR1 and also OPTN have been shown to bind ubiquitin chains on intracellular bacteria and play important roles in directing Ub-coated intracellular bacterial to autophagosome. Many E3 ubiquitin ligases such as RNF166, LRSAM1, Parkin, and Smurf1 have been reported to localize to the bacterial and directly or indirectly ubiquitinate the unknown substrate on its surface. But how OPTN recruits to the ubiquitinated cargos for triggering autophagy is still a mysterious process. Studies on bacteria's ubiquitinated substrates and E3 enzymes may further clarify how OPTN is recruited to bacteria.

2.1.2 Recognition of cargo by OPTN: After recruiting to the cargos, OPTN then bind to the ubiquitinated cargo. Interestingly, OPTN is not bind to all kinds of linkage types of ubiquitin chains randomly, but it have binding preference for linear polyubiquitin chains and K63 chains, rather than K48 or monoubiquitin (Li et al. , 2016). Recent studies with crystal structure analysis reveals an structure of the OPTN UBAN domain in complex with the linear diubiquitin, and phosphorylation modification plays a very critical role in it ( $\mathrm{Li}$ et al. , 2018). On the one hand, phosphorylation of S473 and dual phosphorylation of S473 and S513 in UNAN domain of OPTN by TBK1 enhancing the binding to multiple ubiquitin chains (Heo et al. , 2015). On the other hand, p-S65 UB phosphorylated by PINK1 is lack of attraction to OPTN by GST pull down assay (immunofluorescence of HeLa cells shown the opposite effects), but the loss of binding between OPTN and p-S65 UB can be partially rescued by TBK1 phosphorylated UBAN domain (Heo et al. , 2015). We are closer and closer to the mechanism of recognition of ubiquitin by OPTN and also other autophagy receptors in today's study, why the ubiquitin-modified cargos are selectively recognized by OPTN or other autophagy receptors and how to recognize the cargo efficiently still remain questions that need to be figure out.

\subsection{The Role of OPTN in autophagosome formation}

After binding to ubiquitin decorated cargo through UBD, the OPTN then link cargo to autophagosomal 
membranes via binding to microtubule-associated protein 1 light chain 3( LC3). Until then, autophagosome membrane is needed.

2.2.1 Nucleation of the phagophore: Multiple membrane sources and proteins are required for the nucleation of the phagophore, and OPTN involved in many aspects of the process. OPTN can function by promoting the ULK1 complex (Lazarouet al. , 2015), a very first autophagy-specific complex, translocating to autophagy initiation sites during PINK1/Parkin mitophagy then it drives the downstream activation of the VPS (vacuolar protein sorting) 34 (VPS34) complex, which is served as a second kinase complex. One of the complex members, the phosphoinositide 3-kinase (PI3K), then generates phosphatidylinositol 3-phosphate (PtdIns(3)P), a simple phosphoinositide, which recruits effector proteins WIPI1/2 translocates to PI3P-enriched sites, and Atg12-Atg5-Atg16L1 complex by binding ATG16L1, then conjugates Atg8s onto autophagosomal membranes.

In addition to ULK1 complex recruiting, OPTN can also form a complex with Wipi2 and Atg5-12 conjugate recruiting the autophagy-related Atg12-5-16L1 complex to phagophores containing the Wipi2 protein. Atg12$5-16 \mathrm{~L} 1$ complex is believed to act in covalent modification of LC3 to the phosphatidylethanolamine (PE) in the phagophore membrane (Bansal et al. , 2018). These studies suggest that OPTN might also affect the process of LC3 lipidation. On balance, OPTN affect the initiation and biogenesis of phagophore.

2.2.2 OPTN interact with LC3: Generally, OPTN is well known to bind to Atg8 proteins via the LIR domain and can be enhanced by Ser-177 phosphorylation of OPTN in LIR domain by TBK1 (Wild et al. , 2011). The LIR motif within autophagy receptors is a main feature of selective autophagy models, in which the autophagy receptors mediate the combination of Atg8-associated membranes and the autophagic cargo.

In humans, the Atg8 family consists of six primary members belonging to the LC3 and GABARAP subfamilies. OPTN could interaction with several ATG8 orthologs, LC3A LC3B, and GABARAPL2 to autophagosome maturation. But as a function of autophagy receptor, with LC3C (Verlhac et al. , 2015). As we describe above, OPTN direct the lipidation of LC3 to the phagophore, then the ubiquitinated cargo connected OPTN induces LC3 recruitment via its LIR domain. While, unexpectedly, LIR domain of OPTN might dispensable for LC3 recruitment in mitophagy as F178A mutation of OPTN that lost the LC3 binding ability in LIR domain could still recruit all Atg8s, but reduce the mitophagy efficiency (Padman et al. , 2019). These study is refreshing that both mutation of UBD and LIR domain of OPTN can also be recruited and LIR facilitate in OPTN recruitment as a positive feedback model in an ubiquitin-independent way. The latter model is reasonable, while the role of UBD and LIR domain double-mutated OPTN recruitment is inquisitive. But at the thought of OPTN as a scaffold protein contains several structural domains, nothing is weird at all.

\section{The Role of OPTN in autophagosome maturation}

OPTN may also have important functions in maturation of autophagosome through its interactions with Myosin VI and LC3 family. Autophagosome fusion with the lysosome is a crucial step in autophagy. Myosin VI is currently the only unconventional myosin motor that moves in the opposite direction along actin filaments. In detail, myosin VI, through a direct interaction with $\mathrm{COOH}$-terminal myosin VI-binding domain (aa 412-520) of optineurin, delivers endosomal membranes which is binding with Tom1, a endosomal protein, to autophagosomes, which is required for autophagosome-lysosome fusion. The defective of myosin VI or Tom1 will reduce autophagosomal delivery of endocytic cargo and cause a block in autophagosomes mature to autolysosomes (Tumbarello et al. , 2012). Verlhac et al . further complement and confirm this effect in Maturation of S. Typhimurium-Containing Autophagosomes (Verlhac et al. , 2015). NDP52 as well as OPTN promote autophagosome maturation by interacting with several ATG8 orthologs, such as LC3A, LC3B, and GABARAPL2, but not with LC3C via non-conventional LIR as well as binding MYOSIN VI (Verlhac et al. , 2015). Regarding the maturity of autophagosomes, whether OPTN directly participates in this process or whether the maturation of autophagosomes is affected by the above two steps is worth considering and paying attention.

\subsection{The role of OPTN in lysosome quality control}


Lysosomes are organelles central to degradation and recycling processes in animal cells. In autophagy, autophagosome fuses with a lysosome containing degrading enzymes and leads to the digestion of the autophagosome content. Therefore, although lysosomes often appear as autolysosome in autophagy, the normal function of lysosomes is very important to ensure that autophagy proceeds without a hitch. Claudioet al . using high-resolution and live-cell CLEM in AS-stimulated microglial cells demonstrated that both TBK1 and OPTN are recruitment to ubiquitylated lysosomes to handle with lysosomal quality impairment and restore lysosomal quality control by autophagy, which also called lysophagy named by autophagy substrate(Bussi et al. , 2018). In general, autophagy induction is frequently associated by up-regulated hydrolase synthesis and increased lysosome biogenesis(Ghavami et al. , 2014). When lysosomes are out of stabilizing or their function is impaired, the autophagy inhibitors tend to lessen the stress of autophagic on dysfunctional lysosomes by reducing the delivery of autophagic cargo rather than through an excessively aggressive autophagy process (Ghavami et al. , 2014). Therefore, the normal function of lysosomes is important for autophagy.

\subsection{OPTN as a substrate of autophagy}

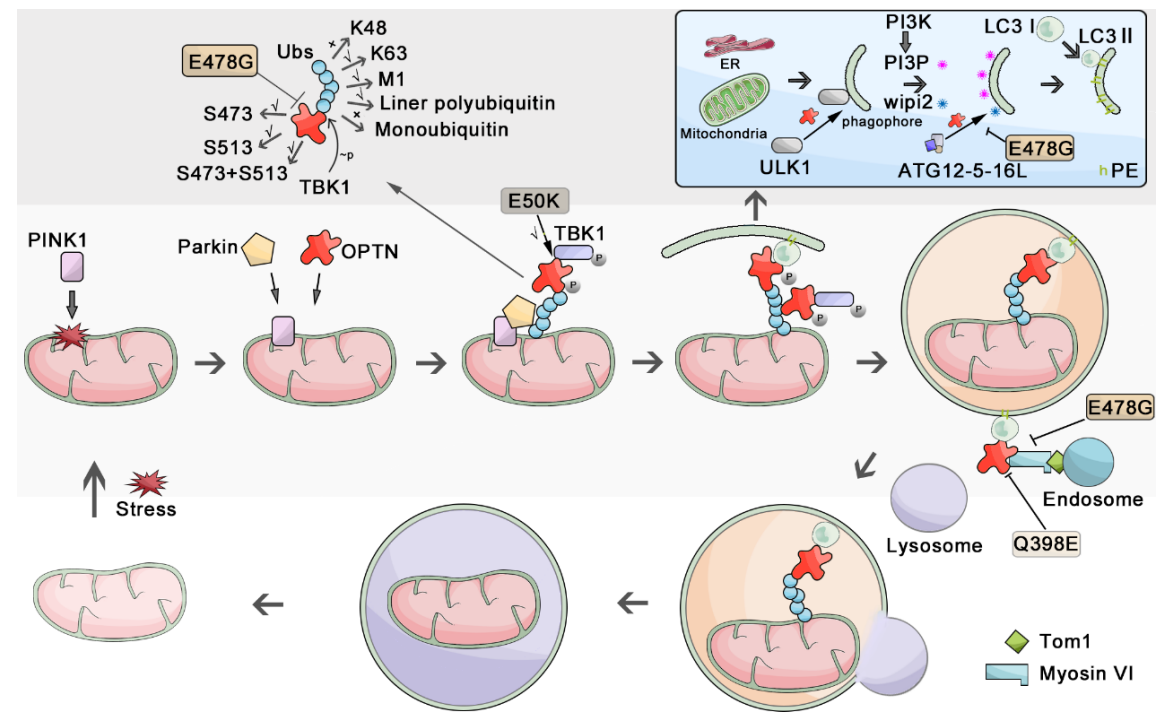

Although, recent study revealed that the UPS (ubiquitin-proteasome system) is the major system for degradation of OPTN, not the autophagy-lysosome pathway (Mao et al. , 2017). But it's obvious that as a participant of autophagy, OPTN itself undergo autophagic degradation, indicating that OPTN not only function as a regulator but also a substrate of autophagy, and recycling to a new OPTN continuing perform autophagic function.

FIGURE 2 The broad role of OPTN in autophagy process

\section{The role of OPTN-mediated autophagy in various diseases}

As we all know, autophagy compromising is associated with countless diseases, such as cancer, neurodegenerative disease, microbial infection and aging, which inspire us that autophagy is a promising target of disease treatment. Interestingly, OPTN dysfunction or mutation are also reported to be closely related to these diseases mentioned above. Understanding the autophagic mechanism in OPTN-dysfunction-associated disease may ultimately allow scientists and clinicians to harness this process for the purpose of improving human health.

\subsection{Glaucoma:}

Glaucoma is the second leading cause of blindness in the world after cataracts (Rezaie et al. , 2002). OPTN was the third genes have been identified linked to Glaucoma by M Sarfarazi group (Rezaie et al. , 2002), 
the other two are $C Y P 1 B 1$, and $M Y O C$. They identified mutations in the coding region of OPTN that were associated with $16.7 \%$ of families with hereditary primary open-angle glaucoma (POAG), the most common type of glaucoma, including individuals with normal intraocular pressure (Rezaie et al. , 2002). This mutations including E50K, 2-base pair (bp) "AG" insertion, Arg545 - Gln (R545Q) and Met98 - Lys (M98K), in which E50K individuals with normal intraocular pressure

OPTN expression is significantly induced after treatment with the factors known to be associated with the disease, such as elevated intraocular pressure (IOP), tumor necrosis factor- $\alpha$ (TNF- $\alpha$ ), and dexamethasone (DEX) (Vittitow et al. , 2002), which further support OPTN is part of the transcriptome responding to the occurrence of glaucoma. Considering the mutation of OPTN would result in Glaucoma, the increased OPTN expression might repose to exert the protective role of this protein in the trabecular meshwork.

So far, a great number of different types of mutations of OPTN discovered in patients of glaucoma have been reported, such as E50K, M98K, H26D, H486R, E322K, etc ., in which E50K and M98K are more frequently and widely studied than others. Different from other mutations including M98K, transgenic mice that expression E50K mutation of OPTN have been developed, obtaining glaucoma phenotype such as loss of RGCs, thinning of various cell layers of retina and gliosis (Menget al. , 2012), suggesting that these are useful as a model of normal-tension glaucoma (NTG), a subgroup of POAG, and also further verification of the occurrence of disease caused by E50K.

Many studies try to elucidate the mechanism of E50K causing Glaucoma. So far, we have hold the evidence that interaction of OPTN with several proteins such as TBK1, Myosin VI and Rab8 will be affected by the mutation of E50K. As a consequence, E50K impairs vesicle trafficking, autophagy, oxidative stress and other alterations. As for oxidative stress, Madhavi et al . suggest that E50K mutant-induced cell death might be mediated by oxidative stress, as NAC and Trolox (two antioxidants) can inhibit E50K induced RGC-5 cell death, and this type of cell death was selectively participated in RGCs rather than in other cells. What's more, overexpressed E50K mutation triggers oxidative stress by increasing ROS production in the retina of aged $\mathrm{E} 50 \mathrm{~K}^{\text {tg }}$ mice (Shim et al. , 2016). But the mechanism of ROS production and upregulated oxidative stress and the connection with E50K induced cell death is yet to be established. Nadia et al. reported that overexpression of OPTN could increases cell survival induced by $\mathrm{H}_{2} \mathrm{O}_{2}$ by translocate to the nucleus in a Rab8 dependent manner and can be disrupted by E50K (De Marco et al. , 2006). This study was conducted in NIH3T3 cells and repeated in a neuronal cell line(Neuro2a), which indicate that changes in biological activity induced by mutation in E50K was pervasive in other cell lines, but the retina might the most affected. Further, E50K mutation of OPTN fails to binding to Rab8, leading to defective anti-oxidative stress induced cell death (Chi et al. , 2010), which provides a possible hypothesis that E50K disrupt the cell protection and nucleus translocation. However, it's still a unsolved puzzle that the role of OPTN in the nucleus in the pathophysiology of Glaucoma.

As for autophagy, many studies have been published but there are some disputes. On the one hand, Shimet al. hold an idea that E50K could induce mitophagy in retinal ganglion cell (RGC). As they observed increased mitophagosome by using TEM in overexpression of E50K in the axons of the glial lamina in $\mathrm{E}^{-} \mathrm{K}^{\text {-tg }}$ mice compare with wild type mice (Shimet al. , 2016; Shim et al. , 2018). What's more, E50K shows the enhanced TBK1 interaction (Li et al. , 2016). As we discussed above, TBK1 could phosphorylate OPTN in S177, S473 and S513, all of which could promote OPTN mediated autophagy by enhance binding activity in LIR and UBD domain. Thus, to some extent, E50K is an mutant that induce autophagy (I don't agree with it). While, on another hand, in a comparison with wild type OPTN, E50K showed a better colocalization with autophagosome, and treatment of E50K transfected cells with Rapamycin, an autophagy activator, inhibiting E50K-induced cell death significantly (Chalasani et al. , 2014). Supposing that E50K could induce autophagy, why would the treatment of Rapamycin could save E50K-induced cell death?

I hold an idea that E50K inhibit autophagy, in other words, E50K inhibit autophagy flux. Nowadays, we have virous assays for monitoring the autophagic process, and we have Guidelines available online with a recommendation that multiple assays to monitor autophagy is needed (Klionsky et al. , 2016). We have showed above in Fig 2, no matter which process is blocked, the autophagy is blocked. While the 
studied conducted by Chim et al. only show the increased number of mitophagosome, the protein level of LC3II as well as an unclear immunohistochemical analysis of LC3. What's more, the increased number of mitochondrial is another evidence that E50K do not induce autophagy. Because no evidence shows the increased mitophagosome is maturing into autolysosome and being degradation in the lysosomal degradation pathway of autophagy. Madhavi et al. using a mCherry-GFP-LC3B plasmids monitoring autophagy by Confocal Microscopy. As the GFP signal quenched in the acidic pH of autolysosomes, so once the mCherryGFP-LC3B goes into autolysosomes, only the red signal can be detected. The results showed that E50K shows the reduced autolysosomes in the amino acid starvation conditions (Chalasani et al. , 2014). The increased interaction with TBK1 and protein level of LC3II might the induced by the fall out of step of E50K mutant, which trigger autophagy on and on with an imperfectly ending. But which step is blocked on earth is unknown. It is worth mentioning that a recent study reported that Glaucoma-mutant OPTN induced Glaucoma is mitophagy independent, which means mitophagy paly a limit role in Glaucoma progression, at least in OPTN-related mutations (Chernyshova et al. , 2019). E50K may affect the maturation of autophagosomes so that the merge with autophagosome is more than WT OPTN (because of the accumulate without being an autolysosome) and its cytotoxicity could be reversed by Rapamycin. These study suggest that there are other proteins that can supplement this function and finding this protein may find new treatment strategies.

\subsection{Amyotrophic lateral sclerosis}

Amyotrophic lateral sclerosis (ALS), which occurs in middle age, is a progressive disease characterized by the degeneration of motor neurons in the primary motor cortex, brain stem and spinal cord. ALS is also a neurodegenerative disorder and little is known about the underlying mechanism of ALS in most patients, but many genes were reported to cause familial ALS (FALS), such as SOD1 (the first ALS gene to be identified in 1993), TBK1 , SQSTM1, etc .(Rosen, 1993; Fecto et al. , 2011; Freischmidt et al. , 2015).In 2010, Maruyama $\mathrm{H}$ et al . identified OPTN as a causative gene for amyotrophic lateral sclerosis for the first time and found three types of mutations: a homozygous deletion of exon 5, a homozygous Q398X nonsense mutation and a heterozygous $\mathrm{E} 478 \mathrm{G}$ missense mutation which is within its ubiquitin-binding domain (Maruyama et al. , 2010). Different from E50K, the role of E478G cause autophagy dysfunction seems none controversial. Before long, another novel missense mutation, Q454E, located in UBD domain of OPTN that causing ALS is reported (van Blitterswijk et al. , 2012). Same as the E478G, Q454E shows impaired mitophagy after CCCP treatment in Hela cells (Chernyshovaet al. , 2019). We focus here on E478G of OPTN, since this is the mutation in UBD domain of OPTN in which autophagy has been most studied. Although the direct connection between ALS and defective autophagy caused by E478G is not confirmed, the E478G mutation severely affects autophagy, which involved in many aspects of autophagy.

Mutation of E478G is located in UBAN domain of OPTN which abolishes the binding to ubiquitin (Wild et al. , 2011). Wonget al . first find that E478G OPTN mutation disrupts autophagosome recruitment which arouse the importance of UBDN domain to OPTN in autophagy. As they find that OPTN siRNAdepleted Hela cell reduce the capacity to recruit LC3 to mitochondria together with the slower rates of autophagosome formation after CCCP treatment (Wong et al. , 2014). A more precise mechanism was reported that the mutation of E478G, as well as Q398X, disrupted the association of OPTN with Myosin VI. As we review above, the association of OPTN and Myosin VI facilitated in autophagosome maturation, which is an indispensable process in autophagy. Similar biological process have also been reported by Bansal et al (Bansal et al. , 2018). They find E478G mutation impairs the ability of OPTN to form autophagosomes probably because of it disrupt the complex formation between OPTN and Atg12-5 conjugate, and then it had an effect in the recruitment of Atg12-5-16L1 complex to Wipi2-positive structures. They also found E478G OPTN mutation showed little co-localization with myosin VI which inhibiting autophagosome-lysosome fusion. Although the mechanism of E478G affecting autophagy has been well studied, there is still no direct evidence that OPTN mutations lead to autophagy defects is a direct factor in the pathogenesis of ALS. In order to figure out the causality, E478G combined with autophagy inducer is worth a try.

In view of current drug therapies for ALS can only offer a slightly clinical benefit for patients and a mild 
improvement in survival, OPTN-targeting drug may be a promising treatment strategy.

\subsection{Cancer}

OPTN is also proved to be a tumor suppressor in cancer. Hu lab first found that OPTN is a tumor suppressor in lung cancer though a HACE1-OPTN axis. In detail, HACE1 could ubiquitylate on Lys193 of OPTN, which is a critical step in HACE1-activated autophagy, and form an autophagic complex with p62, accelerating the total cellular autophagic flux and facilitating in tumor suppression (Liu et al. , 2014). Recently, the Berger group analyzed an overview of the RNA-seq data provided by The Cancer Genome Atlas (TCGA) and found that high expression of the OPTN gene across several tumor types, especially in pancreatic cancer and renal cancer which was associated with the second and first highest OPTN expression of all tumor tissues respectively (Ali et al. , 2019). While they showed a weird phenomenon that OPTN knockdown has limited affect in the proliferation of three different human pancreatic cancer cell lines, Miapaca, BXPC3 and Suit2-007, significantly increased the migration in BXPC3 and Miapaca cells, but strikingly reduced the colony formation in all three cells. Autophagy is considered as a protective biological process in general. While, as for cancer, autophagy is not good for cancer cell, and kills cancer in the cradle (Nassour et al. , 2019). Considering that OPTN could induce autophagy and the Berger group's ambiguous experimental results, we hold the opinion that OPTN is more tend to be a tumor suppressor.

\subsection{Diabetic nephropathy}

In addition, OPTN have antisenescence and protection effect in diabetic nephropathy though enhancing mitophagy. Diabetic nephropathy $((\mathrm{DN})$ is a complication of type 1 diabetes, most frequently and also devastatingly, and its diagnosis is traditionally based on microalbuminuria. As Kehonget al . find in clinical specimens, renal OPTN expression was negative correlation to tubulointerstitial injury scores. OPTN expression is also negatively correlated with serum creatinine level and positively correlated with the estimated glomerular filtration rate (eGFR), which is the indicator of DN. As these relationship turned out, OPTN is closely related to the progression of DN. Further study revealed an interesting result that the renal tubular cells that OPTN-positive ones always without expression senescence marker P16. What's more, overexpression of OPTN increased mitophagosome formation and down regulated P16, P21, SA- $\beta$-gal, and SAHF which are features of cellular senescence and vice versa (Chen et al. , 2018). These study not only verified the role of OPTN in inducing autophagy when overexpression, but also reveal an new role of OPTN in antisenescence in diabetic nephropathy though mitophagy.

\subsection{Paget's disease of bone and Crohn's disease}

OPTN gene constitutes a risk factor for the development of Paget's disease of bone (PDB) and Crohn's disease $(\mathrm{CD})$, both of which are inflammatory diseases. PDB is a common bone disorder characterized by disorganized bone remodeling with the unknown exact cause (Appelman-Dijkstra et al. , 2018). The most likely cause is the infection of chronic paramyxovirus in genetically susceptible people. Although the disease is easily diagnosed and usually effectively treated, the pathogenesis still not fully understood. Thus, it is meaningful to study the pathogenesis of PDB. Genome-wide association studies(GWAS) proved the link between OPTN and PDB is deserved to be mentioned (Albaghaet al. , 2010). GWAS is a new strategy that using the application of millions of single nucleotide polymorphisms (SNPs) in the genome as molecular genetic markers to perform comparison analysis or correlation analysis at the genome-wide level to discover the genetic variations affecting complex traits by comparation (Erdmann et al. , 2018).

Recently, Jennifer lab further confirming the direct contribution of OPTN in PDB progress by successfully construct the $100 \%$ of aged Optn global knockout (Optn ${ }^{-/}$) mice, as the KO mice featuring the key clinical manifestations observed in PDB patients, such as polyostotic osteolytic lesions, mixed-phase lesions, and elevated serum alkaline phosphatase (ALP) levels (Wong et al. , 2020). However, the mechanism is more likely because of the roles of OPTN in modulating NF- $x \mathrm{~B}$ and interferon signaling rather than autophagy at present.

As for CD, the Segal group firstly find macrophage OPTN expression is reduced in a proportion of CD 
patients by microarray analysis. Both OPTN-deficient mice and the OPTN ${ }^{\text {low }}$ patients macrophages secreted reduced levels of TNF- $a$ and IL-6 compared with control after HkEc stimulation (Smith et al. , 2015). The exact cause of the CD is not known, but intracellular bacteria appear to be involved in CD inflammation. Interestingly, OPTN-deficient mice were more susceptible to Citrobacter colitis (Chew et al. , 2015), E. coli peritonitis and Salmonella infection, and Optn-knockdown zebrafish infected with Salmonella also had higher mortality (Chew et al. , 2015; Slowicka et al. , 2016). In intestinal epithelial cells, the impaired autophagy function always results in the more susceptible to intestinal inflammation (Tschurtschenthaler et al. , 2018). What's more, it's widely reported that ubiquitin coated bacteria recruits OPTN and Nemo for xenophagy and local activation of NF- $x \mathrm{~B}$, respectively and Competitively, independently restricting bacterial proliferation (van Wijk et al. , 2017). But the balance of the competitive relationship between OPTN and Nemo for ubiquitin of bacterial is yet to be revealed. It was reported that the deficiency of OPTN would not affected NF- $x$ B activation after the treatment of TNF, LPS, or poly $(\mathrm{I}, \mathrm{C})$ in mice or cells. Thus the role of OPTN-mediated autophagy in CD is already ready to come out.

\subsection{Other diseases}

As mention above, OPTN recognizes substrates mainly through ubiquitin chains. However, there's always an exception, OPTN could facilitate in autophagic clearance of protein aggregates independent of ubiquitination. OPTN could recognize and actively participate in the degradation of htt ex1 Q103 and SOD1 G93C aggregates, models of Huntington's disease (HD) and ALS respectively, through its C-terminal CC domain in a ubiquitin-independent manner (Korac et al. , 2013). This has a enlightenment and reference significance for the role of OPTN in some neurodegenerative diseases. As OPTN is frequently observed in protein inclusions of various neurodegenerative diseases, such as neurons of Parkinson's disease (PD) and neuronal and glial intranuclear of adult-onset neuronal intranuclear inclusion disease patients which might due to the autophagy defective.

\section{Regulation of OPTN and the possibility of it as a therapeutic target against pathologies}

The studies have shown that OPTN participate in autophagy broadly and mutation or depletion of OPTN can cause autophagy dysfunction. Thus, OPTN has certain potential to modulate autophagy. Furthermore, as discussed in this review, defective autophagy induced by OPTN dysfunction may have a strong impact on the course of pathogenies, via disruption of cargo recognition, LC3 interaction, autophagosome formation and maturation, as well as lysosome quality control. In general, OPTN is a promising target for the treatment of autophagy involved disease.

Interestingly, some cytokines derived from immune response, such as TNF- $\alpha$ and IFN- $\gamma$ have been shown to induce expression of OPTN (Sudhakaret al. , 2013; Senoo et al. , 2018). While increases of TNF $\alpha$ in the eye have been associated with glaucomatous conditions, elevated hydrostatic pressure, and retinal degeneration, thus the increased expression of OPTN may be due to disease progression (Vittitow et al. , 2002). Recently, Doaa et al . find Erufosine and LY294002 caused the increased mRNA transcription of OPTN in Miapaca and Suit2-007 cells in a concentration dependently manner (Ali et al. , 2019). LY294002 is a well-known inhibitor of PI3K that can inhibit autophagic sequestration and Erufosine is a excellent inhibitor of mTOR and inducer of LC3B. Therefore, the regulation of OPTN by these two compounds are more likely due to response to autophagy they affect.

There are two proteins reported to regulate OPTN. IFR-1,a critical target and mediator of IFN- $\gamma$ signaling, is reported to be a strong activator of optineurin promoter activity. As we all know, promoters are important factors for gene regulation (Sudhakar et al. , 2013). Therefore, overexpression of IFR-1 might induce OPTN expression level. What's more, figuring out the way of IFR-1 binding with OPTN promotor may give medicinal chemists some ideas to design small molecules that activate OPTN promoter. Furthermore, Hrd1, an E3 ubiquitin ligase, can promote the ubiquitination and degradation of OPTN by the UPS, which can be abolished when the RING finger domain of Hrd1 is absent (Mao et al. , 2017).

So far none drug or small molecule compound are reported can target to OPTN and modulate its expression at either protein or mRNA level. Considering the diverse role in autophagy and disease played by optineurin, 
its tight regulation becomes imperative. Thus, strategies to modulate specifically OPTN with no toxic effects should be developed to establish an efficient therapy for pathogenies treatment.

\section{Conclusion and future perspectives}

Over the past ten years, a great number of studies have shown that the function of OPTN is not simply the autophagy receptor. Indeed, OPTN is gifted and multifunctional in every steps of autophagy process and linked to various diseases. In general, the different sites of mutation of OPTN in different tissues hold the different phenotypes, in others words in human, disorders. However, the pathogenesis of disorders that related to OPTN mutation or dysfunction is not fully understood. To figure it out might good for disease to be effectively treated.

As we reviewed above, OPTN is widely participated in many aspects of autophagy process. Briefly, OPTN can be recruitment around the cargos and then recognize the cargos by specific types of ubiquitin chains. Meanwhile, OPTN located in nucleation site of the phagophore, recruiting the ULK1 complex and triggering nucleation of phagophore as well as facility in the process of Atg8 lipidation in phagophore. After autophagosome established, OPTN delivers endosomal membranes together with some proteins, such as myosin VI and Tom1, and promote autophagosome maturation. What's more, OPTN also play an vital role in lysosome quality control, which guaranteed the autophagy progressing successfully, and degradation with cargos in the autolysosome.

Importantly, OPTN is closely related to many critical disorders. The etiological link between OPTN and ALS, Glaucoma, PBD is tangible. And the etiological link between OPTN and Crohn's disease, diabetic nephropathy and cancer is ambiguous but the relationship of OPTN to these three types of disease is surely close. Autophagy is central to cell homeostasis and impaired autophagy often resulting physiological dysfunction and causing disease. And is widely accepted that autophagy is important and protective in neurodegenerative diseases, inflammatory diseases, and cancer. Thus, inducing autophagy is an effective treatment to these diseases. There are many autophagy activators, such as rapamycin, an mTOR inhibitor, is also an clinical used drug termed sirolimus and for the treatment to acute rejection of renal transplantation patient that can induce autophagy distinctly. However, being an mTOR inhibitor, sirolimus do have many side effects and limiting to therapy (Morath et al. , 2007). Thus, more attentions should paid in safety autophagy activators discovery. OPTN, as an autophagy inducer with no reported cytotoxicity of overexpression and is associated with various diseases, is a promising target of autophagy to treat some of these diseases at least. While, there is no reported chemical compound can induce OPTN directly, but some proteins can enhance the activity or expression of OPTN. Therefore, in the future, efforts should be made in discovering the drug that capacitated in inducing OPTN expression.

\section{References:}

Albagha OM, Visconti MR, Alonso N, Langston AL, Cundy T, Dargie R, et al. (2010). Genome-wide association study identifies variants at CSF1, OPTN and TNFRSF11A as genetic risk factors for Paget's disease of bone. Nature genetics 42: 520-524.

Ali DM, Ansari SS, Zepp M, Knapp-Mohammady M, Berger MR (2019). Optineurin downregulation induces endoplasmic reticulum stress, chaperone-mediated autophagy, and apoptosis in pancreatic cancer cells. Cell death discovery 5: 128.

Appelman-Dijkstra NM, Papapoulos SE (2018). Paget's disease of bone.Best practice 83 research. Clinical endocrinology \& metabolism32: 657-668.

Bansal M, Moharir SC, Sailasree SP, Sirohi K, Sudhakar C, Sarathi DP, et al. (2018). Optineurin promotes autophagosome formation by recruiting the autophagy-related Atg12-5-16L1 complex to phagophores containing the Wipi2 protein. The Journal of biological chemistry293: 132-147.

Bussi C, Peralta Ramos JM, Arroyo DS, Gallea JI, Ronchi P, Kolovou A, et al. (2018). Alpha-synuclein fibrils recruit TBK1 and OPTN to lysosomal damage sites and induce autophagy in microglial cells. Journal 
of cell science 131 .

Chalasani ML, Kumari A, Radha V, Swarup G (2014). E50K-OPTN-induced retinal cell death involves the Rab GTPase-activating protein, TBC1D17 mediated block in autophagy. PloS one 9: e95758.

Chen K, Dai H, Yuan J, Chen J, Lin L, Zhang W, et al. (2018). Optineurin-mediated mitophagy protects renal tubular epithelial cells against accelerated senescence in diabetic nephropathy. Cell death $\mathcal{G}$ disease 9: 105.

Chernyshova K, Inoue K, Yamashita SI, Fukuchi T, Kanki T (2019). Glaucoma-Associated Mutations in the Optineurin Gene Have Limited Impact on Parkin-Dependent Mitophagy. Investigative ophthalmology $\mathscr{E}$ visual science 60: 3625-3635.

Chew TS, O'Shea NR, Sewell GW, Oehlers SH, Mulvey CM, Crosier PS, et al. (2015). Optineurin deficiency in mice contributes to impaired cytokine secretion and neutrophil recruitment in bacteria-driven colitis. Disease models \& mechanisms 8: 817-829.

Chi ZL, Akahori M, Obazawa M, Minami M, Noda T, Nakaya N, et al.(2010). Overexpression of optineurin E50K disrupts Rab8 interaction and leads to a progressive retinal degeneration in mice. Human molecular genetics 19: 2606-2615.

De Marco N, Buono M, Troise F, Diez-Roux G (2006). Optineurin increases cell survival and translocates to the nucleus in a Rab8-dependent manner upon an apoptotic stimulus. Journal of Biological Chemistry281: $16147-16156$.

Erdmann J, Kessler T, Munoz Venegas L, Schunkert H (2018). A decade of genome-wide association studies for coronary artery disease: the challenges ahead. Cardiovascular research 114:1241-1257.

Fecto F, Yan J, Vemula SP, Liu E, Yang Y, Chen W, et al. (2011). SQSTM1 mutations in familial and sporadic amyotrophic lateral sclerosis.Archives of neurology 68: 1440-1446.

Freischmidt A, Wieland T, Richter B, Ruf W, Schaeffer V, Muller K, et al. (2015). Haploinsufficiency of TBK1 causes familial ALS and fronto-temporal dementia. Nature neuroscience 18:631-636.

Ghavami S, Shojaei S, Yeganeh B, Ande SR, Jangamreddy JR, Mehrpour M, et al. (2014). Autophagy and apoptosis dysfunction in neurodegenerative disorders. Progress in neurobiology112: 24-49.

He H, Dang Y, Dai F, Guo Z, Wu J, She X, et al. (2003). Post-translational modifications of three members of the human MAP1LC3 family and detection of a novel type of modification for MAP1LC3B.The Journal of biological chemistry 278: 29278-29287.

Heo JM, Ordureau A, Paulo JA, Rinehart J, Harper JW (2015). The PINK1-PARKIN Mitochondrial Ubiquitylation Pathway Drives a Program of OPTN/NDP52 Recruitment and TBK1 Activation to Promote Mitophagy.Molecular cell 60: 7-20.

Katsuragi Y, Ichimura Y, Komatsu M (2015). p62/SQSTM1 functions as a signaling hub and an autophagy adaptor. The FEBS journal282: 4672-4678.

Klionsky DJ, Abdelmohsen K, Abe A, Abedin MJ, Abeliovich H, Acevedo Arozena A, et al. (2016). Guidelines for the use and interpretation of assays for monitoring autophagy (3rd edition).Autophagy 12: 1-222.

Korac J, Schaeffer V, Kovacevic I, Clement AM, Jungblut B, Behl C, et al. (2013). Ubiquitin-independent function of optineurin in autophagic clearance of protein aggregates. Journal of cell science 126: 580-592.

Lazarou M, Sliter DA, Kane LA, Sarraf SA, Wang C, Burman JL, et al. (2015). The ubiquitin kinase PINK1 recruits autophagy receptors to induce mitophagy. Nature 524: 309-314.

Levine B, Kroemer G (2019). Biological Functions of Autophagy Genes: A Disease Perspective. Cell 176: $11-42$. 
Li F, Xie X, Wang Y, Liu J, Cheng X, Guo Y, et al. (2016). Structural insights into the interaction and disease mechanism of neurodegenerative disease-associated optineurin and TBK1 proteins. Nature communications 7: 12708 .

Li FX, Xu DC, Wang YL, Zhou ZX, Liu JP, Hu SC, et al. (2018). Structural insights into the ubiquitin recognition by OPTN (optineurin) and its regulation by TBK1-mediated phosphorylation. Autophagy14: 66-79.

Li Y, Kang J, Horwitz MS (1998). Interaction of an adenovirus E3 14.7-kilodalton protein with a novel tumor necrosis factor alpha-inducible cellular protein containing leucine zipper domains.Molecular and cellular biology 18: 1601-1610.

Liu Z, Li H, Hong C, Chen M, Yue T, Chen C, et al. (2018). ALS-Associated E478G Mutation in Human OPTN (Optineurin) Promotes Inflammation and Induces Neuronal Cell Death. Frontiers in immunology 9: 2647.

Liu Z, Chen P, Gao H, Gu Y, Yang J, Peng H, et al. (2014). Ubiquitylation of autophagy receptor Optineurin by HACE1 activates selective autophagy for tumor suppression. Cancer cell26: 106-120.

Mao J, Xia Q, Liu C, Ying Z, Wang H, Wang G (2017). A critical role of Hrd1 in the regulation of optineurin degradation and aggresome formation. Human molecular genetics 26: 1877-1889.

Maruyama H, Morino H, Ito H, Izumi Y, Kato H, Watanabe Y, et al.(2010). Mutations of optineurin in amyotrophic lateral sclerosis. Nature 465: 223-226.

Meng Q, Xiao Z, Yuan H, Xue F, Zhu Y, Zhou X, et al. (2012). Transgenic mice with overexpression of mutated human optineurin(E50K) in the retina. Molecular biology reports 39: 1119-1124.

Morath C, Arns W, Schwenger V, Mehrabi A, Fonouni H, Schmidt J, et al. (2007). Sirolimus in renal transplantation. Nephrology, dialysis, transplantation : official publication of the European Dialysis and Transplant Association - European Renal Association 22 Suppl 8: viii61-viii65.

Nakatogawa H, Suzuki K, Kamada Y, Ohsumi Y (2009). Dynamics and diversity in autophagy mechanisms: lessons from yeast. Nature reviews. Molecular cell biology 10: 458-467.

Nassour J, Radford R, Correia A, Fuste JM, Schoell B, Jauch A, et al. (2019). Autophagic cell death restricts chromosomal instability during replicative crisis. Nature 565: 659-663.

Padman BS, Nguyen TN, Uoselis L, Skulsuppaisarn M, Nguyen LK, Lazarou M (2019). LC3/GABARAPs drive ubiquitin-independent recruitment of Optineurin and NDP52 to amplify mitophagy. Nature communications 10: 408.

Park BC, Shen X, Samaraweera M, Yue BY (2006). Studies of optineurin, a glaucoma gene: Golgi fragmentation and cell death from overexpression of wild-type and mutant optineurin in two ocular cell types. The American journal of pathology 169: 1976-1989.

Pohl C, Dikic I (2019). Cellular quality control by the ubiquitin-proteasome system and autophagy. Science $366: 818-822$.

Rezaie T, Sarfarazi M (2005). Molecular cloning, genomic structure, and protein characterization of mouse optineurin. Genomics85: 131-138.

Rezaie T, Child A, Hitchings R, Brice G, Miller L, Coca-Prados M, et al. (2002). Adult-onset primary open-angle glaucoma caused by mutations in optineurin. Science 295: 1077-1079.

Rosen DR (1993). Mutations in $\mathrm{Cu} / \mathrm{Zn}$ superoxide dismutase gene are associated with familial amyotrophic lateral sclerosis. Nature $364: 362$. 
Schwamborn K, Weil R, Courtois G, Whiteside ST, Israel A (2000). Phorbol esters and cytokines regulate the expression of the NEMO-related protein, a molecule involved in a NF-kappa B-independent pathway. The Journal of biological chemistry 275: 22780-22789.

Senoo K, Yamashiro K, Yamamoto T, Myokai F, Kawamura M, Takashiba S (2018). Expression of optineurin isolated from rat-injured dental pulp and the effects on inflammatory signals in normal rat kidney cells. Odontology 106: 135-144.

Shim MS, Takihara Y, Kim KY, Iwata T, Yue BY, Inatani M, et al.(2016). Mitochondrial pathogenic mechanism and degradation in optineurin E50K mutation-mediated retinal ganglion cell degeneration.Scientific reports 6: 33830 .

Shim MS, Kim KY, Noh M, Ko JY, Ahn S, An MA, et al. (2018). Optineurin E50K triggers BDNF deficiency-mediated mitochondrial dysfunction in retinal photoreceptor cell line. Biochemical and biophysical research communications 503: 2690-2697.

Slowicka K, Vereecke L, Mc Guire C, Sze M, Maelfait J, Kolpe A, et al. (2016). Optineurin deficiency in mice is associated with increased sensitivity to Salmonella but does not affect proinflammatory NF-kappaB signaling. European journal of immunology 46: 971-980.

Smith AM, Sewell GW, Levine AP, Chew TS, Dunne J, O'Shea NR, et al. (2015). Disruption of macrophage pro-inflammatory cytokine release in Crohn's disease is associated with reduced optineurin expression in a subset of patients. Immunology 144: 45-55.

Sudhakar C, Vaibhava V, Swarup G (2013). IRF-1-binding site in the first intron mediates interferon-gammainduced optineurin promoter activation.Biochemical and biophysical research communications 437:179-184.

Tschurtschenthaler M, Adolph TE (2018). The Selective Autophagy Receptor Optineurin in Crohn's Disease. Frontiers in immunology9: 766 .

Tumbarello DA, Waxse BJ, Arden SD, Bright NA, Kendrick-Jones J, Buss F (2012). Autophagy receptors link myosin VI to autophagosomes to mediate Tom1-dependent autophagosome maturation and fusion with the lysosome. Nature cell biology 14: 1024-1035.

Tumbarello DA, Manna PT, Allen M, Bycroft M, Arden SD, Kendrick-Jones J, et al. (2015). The Autophagy Receptor TAX1BP1 and the Molecular Motor Myosin VI Are Required for Clearance of Salmonella Typhimurium by Autophagy. PLoS pathogens 11: e1005174.

van Blitterswijk M, van Vught PW, van Es MA, Schelhaas HJ, van der Kooi AJ, de Visser M, et al. (2012). Novel optineurin mutations in sporadic amyotrophic lateral sclerosis patients. Neurobiology of aging 33: 1016 e1011-1017.

van Wijk SJL, Fricke F, Herhaus L, Gupta J, Hotte K, Pampaloni F, et al. (2017). Linear ubiquitination of cytosolic Salmonella Typhimurium activates NF-kappaB and restricts bacterial proliferation. Nature microbiology 2: 17066 .

Verlhac P, Gregoire IP, Azocar O, Petkova DS, Baguet J, Viret C, et al. (2015). Autophagy receptor NDP52 regulates pathogen-containing autophagosome maturation. Cell host $\& 3$ microbe 17:515-525.

Vittitow JL, Borras T (2002). Expression of optineurin, a glaucoma-linked gene, is influenced by elevated intraocular pressure. Biochemical and biophysical research communications 298:67-74.

Weil R, Laplantine E, Curic S, Genin P (2018). Role of Optineurin in the Mitochondrial Dysfunction: Potential Implications in Neurodegenerative Diseases and Cancer. Frontiers in immunology 9: 1243.

Wen X, Klionsky DJ (2016). An overview of macroautophagy in yeast.Journal of molecular biology 428: 1681-1699. 
Wild P, Farhan H, McEwan DG, Wagner S, Rogov VV, Brady NR, et al.(2011). Phosphorylation of the autophagy receptor optineurin restricts Salmonella growth. Science 333: 228-233.

Wong SW, Huang BW, Hu X, Ho Kim E, Kolb JP, Padilla RJ, et al.(2020). Global deletion of Optineurin results in altered type I IFN signaling and abnormal bone remodeling in a model of Paget's disease.Cell death and differentiation 27: 71-84.

Wong YC, Holzbaur EL (2014). Optineurin is an autophagy receptor for damaged mitochondria in parkinmediated mitophagy that is disrupted by an ALS-linked mutation. Proceedings of the National Academy of Sciences of the United States of America 111: E4439-4448.

Xin Y, Yu L, Chen Z, Zheng L, Fu Q, Jiang J, et al. (2001). Cloning, expression patterns, and chromosome localization of three human and two mouse homologues of GABA(A) receptor-associated protein.Genomics 74: 408-413.

Ying H, Yue BY (2016). Optineurin: The autophagy connection. Experimental eye research 144: 73-80.

Zaffagnini G, Martens S (2016). Mechanisms of Selective Autophagy.Journal of molecular biology 428: 1714-1724.

Zhu G, Wu CJ, Zhao Y, Ashwell JD (2007). Optineurin negatively regulates TNFalpha- induced NF-kappaB activation by competing with NEMO for ubiquitinated RIP. Current biology : CB 17: 1438-1443.
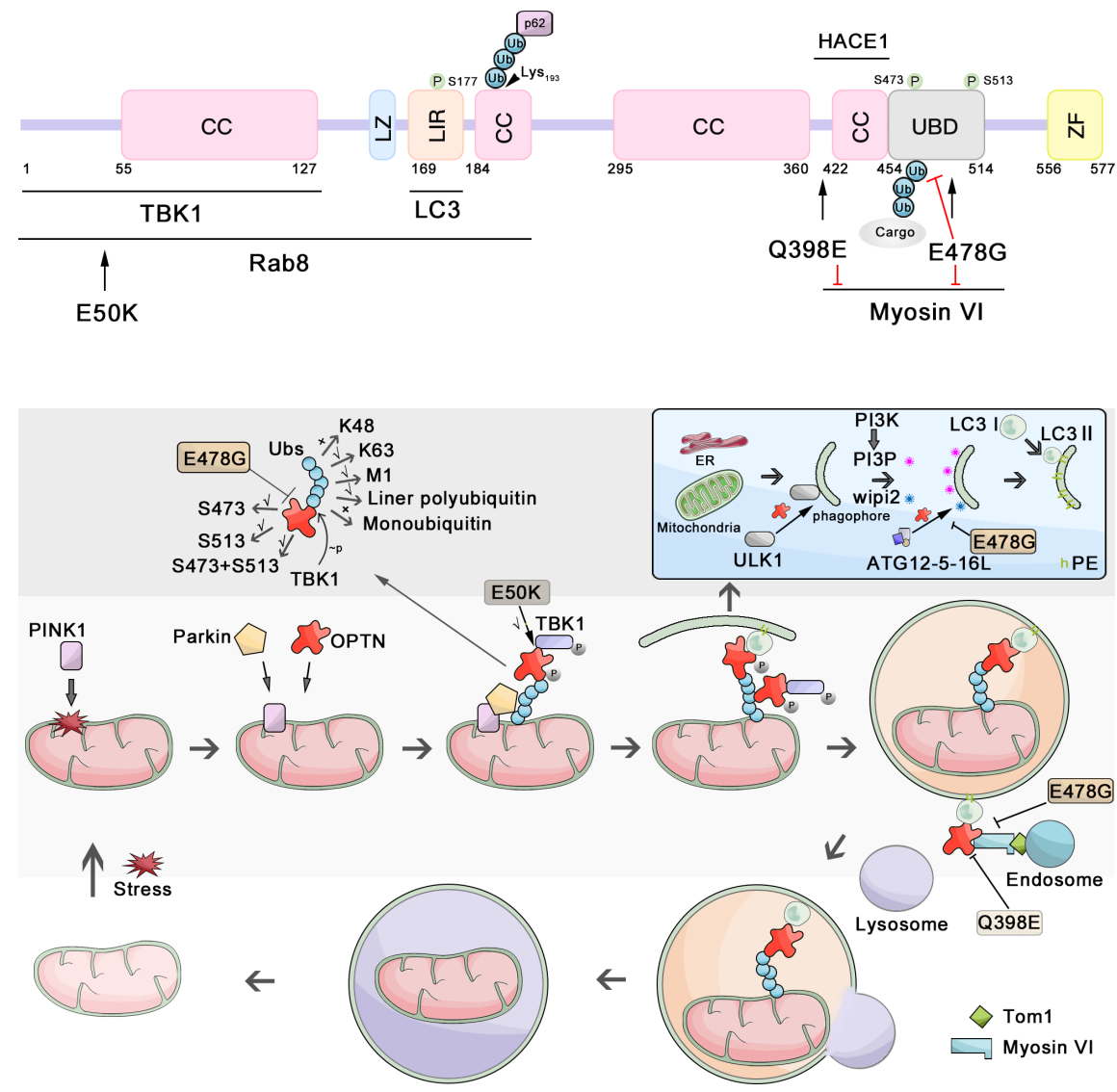